\title{
$\beta$-Decay Half-Lives of ${ }^{76,77} \mathrm{Co},{ }^{79,80} \mathrm{Ni}$, and ${ }^{81} \mathrm{Cu}$ : Experimental Indication of a Doubly Magic ${ }^{78} \mathrm{Ni}$
}

Z. Y. Xu, ${ }^{1,2, *}$ S. Nishimura, ${ }^{2}$ G. Lorusso, ${ }^{2}$ F. Browne, ${ }^{3,2}$ P. Doornenbal, ${ }^{2}$ G. Gey, ${ }^{4,5,2}$ H.-S. Jung, ${ }^{6}$ Z. Li, ${ }^{7}$ M. Niikura, ${ }^{1}$ P.-A. Söderström, ${ }^{2}$ T. Sumikama, ${ }^{8}$ J. Taprogge, ${ }^{9,10,2}$ Zs. Vajta, ${ }^{11,2}$ H. Watanabe,${ }^{12}$ J. Wu, ${ }^{7,2}$ A. Yagi, ${ }^{13}$ K. Yoshinaga, ${ }^{14}$ H. Baba, ${ }^{2}$ S. Franchoo, ${ }^{15}$ T. Isobe, ${ }^{2}$ P. R. John, ${ }^{16}$ I. Kojouharov, ${ }^{17}$ S. Kubono, ${ }^{2}$ N. Kurz, ${ }^{17}$ I. Matea,${ }^{15}$ K. Matsui, ${ }^{1}$ D. Mengoni, ${ }^{16}$ P. Morfouace, ${ }^{15}$ D. R. Napoli, ${ }^{18}$ F. Naqvi, ${ }^{19, \dagger}$ H. Nishibata, ${ }^{13}$ A. Odahara, ${ }^{13}$ E. Şahin, ${ }^{20}$ H. Sakurai, ${ }^{1,2}$ H. Schaffner, ${ }^{17}$ I. G. Stefan, ${ }^{15}$ D. Suzuki, ${ }^{15}$ R. Taniuchi, ${ }^{1}$ and V. Werner ${ }^{19, \$}$

${ }^{1}$ Department of Physics, University of Tokyo, Hongo 7-3-1, Bunkyo-ku, 113-0033 Tokyo, Japan

${ }^{2}$ RIKEN Nishina Center, 2-1 Hirosawa, Wako, Saitama 351-0198, Japan

${ }^{3}$ School of Computing, Engineering and Mathematics, University of Brighton, Brighton BN2 4GJ, United Kingdom

${ }^{4}$ LPSC, Université Grenoble-Alpes, CNRS/IN2P3, F-38026 Grenoble Cedex, France

${ }^{5}$ ILL, 38042 Grenoble Cedex, France

${ }^{6}$ Department of Physics, University of Notre Dame, Notre Dame, Indiana 46556, USA

${ }^{7}$ Department of Physics, Peking University, Beijing 100871, China

${ }^{8}$ Department of Physics, Tohoku University, 6-3 Aramaki-Aoba, Aoba, Sendai, Miyagi 980-8578, Japan

${ }^{9}$ Departamento de Física Teórica, Universidad Autónoma de Madrid, E-28049 Madrid, Spain

${ }^{10}$ Instituto de Estructura de la Materia, CSIC, E-28006 Madrid, Spain

${ }^{11}$ Institute for Nuclear Research, Hungarian Academy of Sciences, P.O. Box 51, Debrecen H-4001, Hungary

${ }^{12}$ IRCNPC, School of Physics and Nuclear Energy Engineering, Beihang University, Beijing 100191, China

${ }^{13}$ Department of Physics, Osaka University, Machikaneyama 1-1, Toyonaka, Osaka 560-0043, Japan

${ }^{14}$ Department of Physics, Tokyo University of Science, 2641 Yamazaki, Noda, Chiba 278-8510, Japan

${ }^{15}$ Institut de Physique Nucléaire d'Orsay, IN2P3-CNRS, F-91406 Orsay, France

${ }^{16}$ Dipartimento di Fisica e Astronomia, Universitá di Padova and INFN Sezione di Padova, I-35131 Padova, Italy

${ }^{17}$ GSI Helmholtzzentrum für Schwerionenforschung GmbH, 64291 Darmstadt, Germany

${ }^{18}$ Istituto Nazionale di Fisica Nucleare, Laboratori Nazionali di Legnaro, I-35020 Legnaro, Italy

${ }^{19}$ Wright Nuclear Structure Laboratory, Yale University, New Haven, Connecticut 06511, USA

${ }^{20}$ Department of Physics, University of Oslo, Oslo NO-0316, Norway

(Received 28 March 2014; published 16 July 2014)

The half-lives of 20 neutron-rich nuclei with $Z=27-30$ have been measured at the RIBF, including five new half-lives of ${ }^{76} \mathrm{Co}\left(21.7_{-4.9}^{+6.5} \mathrm{~ms}\right),{ }^{77} \mathrm{Co}\left(13.0_{-4.3}^{+7.2} \mathrm{~ms}\right),{ }^{79} \mathrm{Ni}\left(43.0_{-7.5}^{+8.6} \mathrm{~ms}\right),{ }^{80} \mathrm{Ni}\left(23.9_{-17.2}^{+26.0} \mathrm{~ms}\right)$, and ${ }^{81} \mathrm{Cu}(73.2 \pm 6.8 \mathrm{~ms})$. In addition, the half-lives of ${ }^{73-75} \mathrm{Co},{ }^{74-78} \mathrm{Ni},{ }^{78-80} \mathrm{Cu}$, and ${ }^{80-82} \mathrm{Zn}$ were determined with higher precision than previous works. Based on these new results, a systematic study of the $\beta$-decay half-lives has been carried out, which suggests a sizable magicity for both the proton number $Z=28$ and the neutron number $N=50$ in ${ }^{78} \mathrm{Ni}$.

DOI: 10.1103/PhysRevLett.113.032505

PACS numbers: 23.40.-s, 27.50.+e

Atomic nuclei are quantum many-body systems consisting of two distinct types of fermions-protons and neutrons. Analogous to atomic physics, the concept of nuclear shell structure was triggered by the discovery of particularly stable nuclei with specific numbers of proton and neutron, such as $2,8,20,28,50,82$, and 126 along the $\beta$-stability line [1]. By assuming a strong spin-orbit interaction within a mean field potential, these magic numbers were correctly interpreted and regarded to be immutable throughout the nuclear chart $[2,3]$. However, with the development of experimental techniques exploiting radioactive ion beams, many nuclei with extreme neutron-to-proton ratios $(N / Z)$, so-called exotic nuclei, have been produced and studied in the last few decades. The results obtained heretofore have demonstrated that the shell structure established for nuclei near the $\beta$-stability line may change drastically in these exotic nuclei. For instance, classical magic numbers in ${ }^{12} \mathrm{Be}(N=8),{ }^{32} \mathrm{Mg}(N=20)$, and ${ }^{42} \mathrm{Si}(N=28)$ were found to disappear [4-6], whereas new magic numbers emerged in ${ }^{24} \mathrm{O}(N=16)$ and ${ }^{54} \mathrm{Ca}$ $(N=34)$ [7-9]. To address the origins of shell evolution in heavier mass regions, it is of particular interest to investigate the properties of nuclei in the vicinity of ${ }^{78} \mathrm{Ni}$, which has the proton number $Z=28$ and the neutron number $N=50$ with a large neutron excess $N / Z \approx 1.8$.

To study the shell evolution around ${ }^{78} \mathrm{Ni}$, many experimental efforts have been made. One of the interesting phenomena related to the proton $Z=28$ shell gap is the monopole migration in $\mathrm{Cu}$ isotopes. A sudden drop of the excited $5 / 2^{-}$state relative to the ground $3 / 2^{-}$state was observed in ${ }^{71,73} \mathrm{Cu}[10,11]$. These two states are characterized by a single-particle nature [12] and their order was 
found to be transposed in ${ }^{75} \mathrm{Cu}$ compared with ${ }^{71,73} \mathrm{Cu}$ [13]. This phenomenon can be understood in terms of a strong tensor interaction, which is attractive between the neutron $\nu g_{9 / 2}$ single-particle orbit (SPO) and proton $\pi f_{5 / 2}$ SPO and repulsive between the $\nu g_{9 / 2}$ and $\pi f_{7 / 2}$ SPOs [14]. The overall effect is to reduce the proton $Z=28$ energy gap between the $\pi f_{7 / 2}$ and $\pi f_{5 / 2}$ SPOs when neutrons start filling the $\nu g_{9 / 2} \mathrm{SPO}$ and reach its maximum at $N=50$, leading to a quenched $Z=28$ shell gap in ${ }^{78} \mathrm{Ni}$ [15]. Conversely, the evolution of the neutron $N=50$ shell closure has been established down to $Z=30$ by nuclear mass measurements. The corresponding two-neutron separation energies show a decreasing $N=50$ shell gap from $Z=40$ to $Z=32$ and a subsequent increase in $Z=30,31$ $[16,17]$. If the increasing trend observed below $Z=32$ was retained, an enhanced $N=50$ shell-gap energy relative to neighboring isotopic chains is expected at $Z=28$, forming a robust neutron shell closure in ${ }^{78} \mathrm{Ni}[16,18]$. Despite these extensive studies, the doubly magic character of ${ }^{78} \mathrm{Ni}$ has not been verified experimentally, especially for the proton shell magicity in ${ }^{78} \mathrm{Ni}$.

Besides the excitation energies and nuclear masses, it is also possible to infer the presence of shell gaps solely from $\beta$-decay half-lives [19]. The first half-life measurement for ${ }^{78} \mathrm{Ni}$ was performed in 2005 [20], and several half-lives have been reported on the lower $N / Z$ side of ${ }^{78} \mathrm{Ni}$ [21-25]. This Letter presents new half-lives beyond ${ }^{78} \mathrm{Ni}:{ }^{76,77} \mathrm{Co}$, ${ }^{79,80} \mathrm{Ni}$, and ${ }^{81} \mathrm{Cu}$. In addition, the half-lives of ${ }^{72-75} \mathrm{Co}$, ${ }^{74-78} \mathrm{Ni},{ }^{78-80} \mathrm{Cu}$, and ${ }^{80-82} \mathrm{Zn}$ were remeasured to a higher precision than in previous works.

The experiment was performed at the RIBF facility in RIKEN. A high-intensity ${ }^{238} \mathrm{U}$ beam was accelerated up to an energy of $345 \mathrm{~A} \mathrm{MeV}$ by the RIKEN cyclotron accelerator complex before impinging on a 3-mm-thick beryllium target to produce secondary beams via in-flight fission. The ${ }^{238} \mathrm{U}^{86+}$ beam was delivered at an average current of $5 \mathrm{pnA}$ at the production target position. The fission products were separated based on their momenta and mass-to-charge ratios $(A / Q)$ by the first stage of BigRIPS in conjunction with a 6-mm-thick $\mathrm{Al}$ achromatic energy degrader placed at the dispersive focal plane [26]. Then, an event-by-event particle identification (PID) was carried out via the $\Delta E-B \rho-\mathrm{TOF}$ method, in which the energy loss, the magnetic rigidity, and the time of flight were measured using the combination of the second stage of BigRIPS and the ZeroDegree Spectrometer (ZDS), giving $Z$ and $A / Q$ information for all the fragments passing through the beam line [27]. The resultant PID plot is presented in Fig. 1. During the 13 days of beam time, about $1.2 \times 10^{4}{ }^{78} \mathrm{Ni}$ nuclei were identified and delivered to the experimental decay station at the end of the ZDS. The highly segmented beam stopper, wide-range active silicon strip stopper array for beta and ion detection (WAS3ABi), was composed of eight double-sided silicon strip detectors and dedicated to the implantation of heavy ions and the

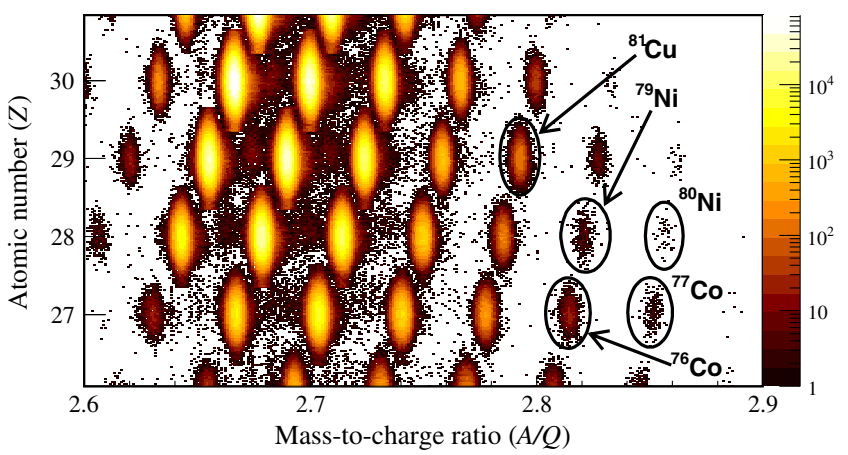

FIG. 1 (color online). PID plot of the experiment. The nuclei with new half-lives determined in this work are highlighted by black circles. The identification of the new isotopes ${ }^{77} \mathrm{Co}$ and ${ }^{80} \mathrm{Ni}$ is reported in Ref. [28].

detection of their $\beta$-decay electrons [29]. Each silicon detector had a $1 \mathrm{~mm}$ thickness and an active area of $60 \times 40 \mathrm{~mm}^{2}$, which was segmented into 60 strips horizontally and 40 strips vertically. The $\gamma$-ray detector, EUROBALL-RIKEN Cluster Array (EURICA) [30,31], was mounted surrounding WAS3ABi to detect $\beta$-delayed $\gamma$ rays as well as $\gamma$ rays emitted by implanted isomers.

Decay events were associated to heavy-ion implantations based on position and time information from the active stopper, and decay curves of different nuclei were constructed as a time difference between implantation and correlated $\beta$ decays. The half-lives of ${ }^{77} \mathrm{Co},{ }^{79,80} \mathrm{Ni}$, and ${ }^{81} \mathrm{Cu}$ were deduced by fitting the decay curves with an unbinned maximum likelihood (MLH) method, which accounted for the activities of parent, daughter, and granddaughter nuclei including $\beta$-delayed neutron-emission $(\beta n)$ channels. Half-lives of decay products and known $\beta n$ probabilities $\left(P_{n}\right)$ were taken from literature, whereas experimentally unknown $P_{n}$ values were estimated by comprehensively considering the global calculations of Möller et al. [32] and Borzov [33] together with a conservative $\pm 25 \%$ uncertainty. The systematic errors introduced by $P_{n}$ values and known half-lives were combined with the statistical error, for which the statistical error was the predominant contribution to the total experimental uncertainty of this work. An example of the MLH fitting analysis, including the constructed decay curve and fitting function, is presented for ${ }^{79} \mathrm{Ni}$ in Fig. 2. In contrast, the $\beta$-electron detections in coincidence with the $\gamma$ decays in daughter nuclei were carried out for the nuclei ${ }^{72-76} \mathrm{Co}$, ${ }^{74-78} \mathrm{Ni},{ }^{78-80} \mathrm{Cu}$, and ${ }^{80-82} \mathrm{Zn}$ to determine half-lives from decay curves including solely the activities of parent nuclei. A detailed description of the analysis procedure can be found in Ref. [34]. The half-lives $T_{1 / 2}^{\exp }$ obtained in the present work are listed in Table I and compared with literature values $T_{1 / 2}^{\mathrm{lit}}$. It is noted that most of the half-lives deduced in this work are in good agreement with literature values except for ${ }^{72} \mathrm{Co}$ and ${ }^{82} \mathrm{Zn}$, which deviate more than $\pm 2 \sigma$ from the previous measurements $[24,35]$. 


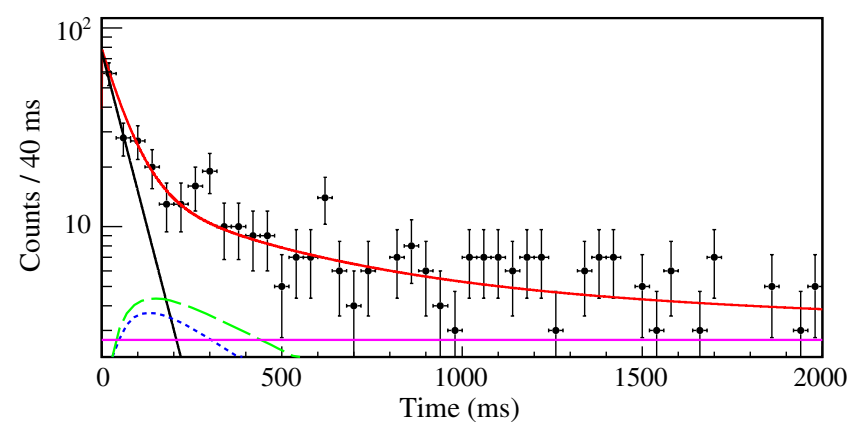

FIG. 2 (color online). Time distribution of the $\beta$-decay events correlated with implanted ${ }^{79} \mathrm{Ni}$. The fitting function (solid red line) considers the activities of parent nuclei (dashed-dotted black line), $\beta$-decay daughter nuclei (fine-dashed blue line), $\beta n$-decay daughter nuclei (dashed green line), a constant background (solid pink line), and other decay products (granddaughter nuclei, etc.), which are not drawn in the figure.

Figure 3 shows the systematic trend of $\beta$-decay half-lives for the nuclei around ${ }^{78} \mathrm{Ni}$ as a function of neutron number. As discussed in Ref. [36], when $\beta$-decay $Q_{\beta}$ values are sufficiently large, the half-life is dominated by a fifth-power dependence on $Q_{\beta}$. In this case, a linear relationship between the $\log _{10} T_{1 / 2}$ and the neutron number of the parent nucleus is expected phenomenologically when $Q_{\beta}$ evolves smoothly in an isotopic chain. Experimentally, this linearity clearly emerges in Fig. 3 below $N=50$. Beyond that, a sudden reduction manifests in the $Z=28$ isotopic chain due to the shorter half-lives of ${ }^{79,80} \mathrm{Ni}$ with reference to the systematics at $N \leq 50$. Since no strong deformation and shape transition were expected in this region, the fast $\beta$-decay processes in ${ }^{79,80} \mathrm{Ni}$ could be attributed to the first neutron outside the $N=50$ shell as follows. If a large shell gap existed, the neutron outside the shell can dramatically increase the $Q_{\beta}$ values and $\beta$-decay rates of ${ }^{79,80} \mathrm{Ni}$ compared to that of ${ }^{78} \mathrm{Ni}$, even though the possible decay

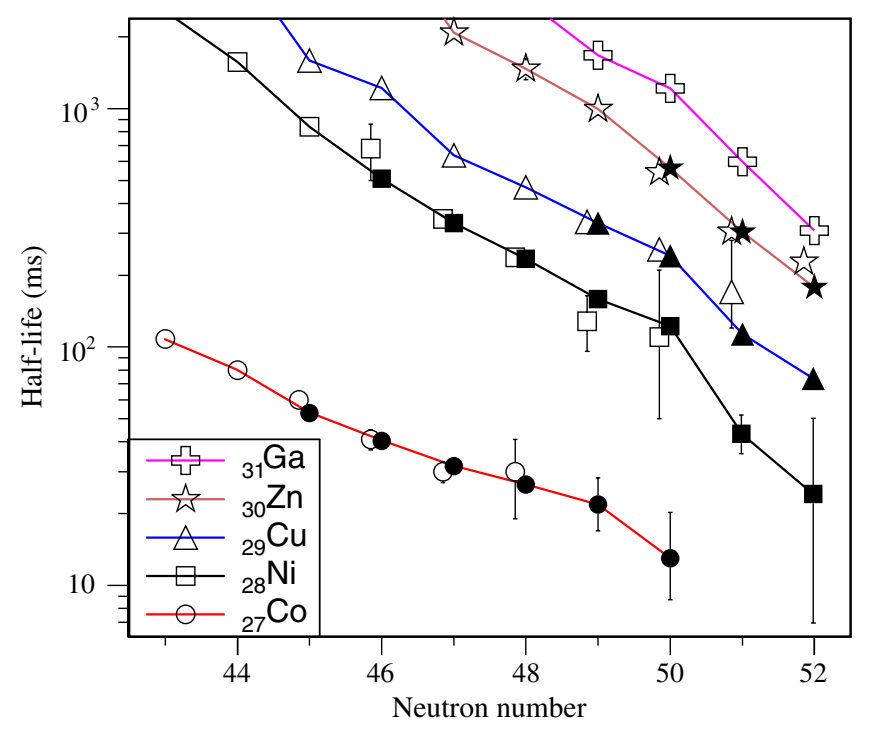

FIG. 3 (color online). Experimental half-lives as a function of neutron number for the isotopes with $Z=27-31$. All the solid symbols represent the half-lives determined by this work while the open symbols are the half-lives taken from Refs. [21,23,24,35]. The systematic trends in different isotopic chains are highlighted by lines connecting the data points with a smaller uncertainty.

channels such as $\nu d_{5 / 2} \rightarrow \pi f_{5 / 2}$ or $\nu s_{1 / 2} \rightarrow \pi p_{3 / 2,1 / 2}$ belong to first-forbidden (FF) transitions. Note that this sudden shortening of the half-lives beyond $N=50$ is not clearly observed in the $\mathrm{Zn}$ and $\mathrm{Ga}$ isotopic chains, and the kink shown by the half-lives of ${ }^{78-81} \mathrm{Cu}$ is much weaker than that in the $\mathrm{Ni}$ isotopes. Since the $Q_{\beta}$ variation crossing the $N=50$ shell is determined by the strength of the neutron shell gap, the stronger kink shown in the $\mathrm{Ni}$ isotopic chain is in compliance with the predicted enhancement of the $N=50$ shell at $Z=28$ : although the calculated neutron shell-gap energies diverge from 2 to $7 \mathrm{MeV}$ in

TABLE I. Beta-decay half-lives obtained in the present work $\left(T_{1 / 2}^{\exp }\right)$ together with the compared literature values $\left(T_{1 / 2}^{\mathrm{lit}}\right)$.

\begin{tabular}{lccccc}
\hline \hline Nuclide & $T_{1 / 2}^{\text {exp }}(\mathrm{ms})$ & $T_{1 / 2}^{\mathrm{lit}}(\mathrm{ms})$ & Nuclide & $T_{1 / 2}^{\text {exp }}(\mathrm{ms})$ & $T_{1 / 2}^{\text {lit }}(\mathrm{ms})$ \\
\hline${ }^{72} \mathrm{Co}$ & $52.8 \pm 1.6$ & $59.9 \pm 1.7^{\mathrm{a}}$ & ${ }^{78} \mathrm{Ni}$ & $122.2 \pm 5.1$ & $110_{-60}^{+100 \mathrm{a}}$ \\
${ }^{73} \mathrm{Co}$ & $40.4 \pm 1.3$ & $41 \pm 4^{\mathrm{a}}$ & ${ }^{79} \mathrm{Ni}$ & $43.0_{-75}^{+8.6}$ & $\cdots$ \\
${ }^{74} \mathrm{Co}$ & $31.6 \pm 1.5$ & $30 \pm 3^{\mathrm{b}}$ & ${ }^{80} \mathrm{Ni}$ & $23.9_{-17.2}^{+26.0}$ & $\cdots$ \\
${ }^{75} \mathrm{Co}$ & $26.5 \pm 1.2$ & $30 \pm 11^{\mathrm{c}}$ & ${ }^{78} \mathrm{Cu}$ & $330.7 \pm 2.0$ & $335 \pm 11^{\mathrm{a}}$ \\
${ }^{76} \mathrm{Co}$ & $21.7_{-4.9}^{+6.5}$ & $\cdots$ & ${ }^{79} \mathrm{Cu}$ & $241.3 \pm 2.1$ & $257_{-26}^{+29 \mathrm{c}}$ \\
${ }^{77} \mathrm{Co}$ & $13.0_{-4.3}^{+7.2}$ & $\cdots$ & ${ }^{80} \mathrm{Cu}$ & $113.3 \pm 6.4$ & $170_{-50}^{+110 \mathrm{a}}$ \\
${ }^{74} \mathrm{Ni}$ & $507.7 \pm 4.6$ & $680 \pm 180^{\mathrm{a}}$ & ${ }^{81} \mathrm{Cu}$ & $73.2 \pm 6.8$ & $\cdots$ \\
${ }^{75} \mathrm{Ni}$ & $331.6 \pm 3.2$ & $344 \pm 25^{\mathrm{a}}$ & ${ }^{80} \mathrm{Zn}$ & $562.2 \pm 3.0$ & $540 \pm 20^{\mathrm{a}}$ \\
${ }^{76} \mathrm{Ni}$ & $234.6 \pm 2.7$ & $238 \pm 18^{\mathrm{c}}$ & ${ }^{81} \mathrm{Zn}$ & $303.2 \pm 2.6$ & $304 \pm 13^{\mathrm{a}}$ \\
${ }^{77} \mathrm{Ni}$ & $158.9 \pm 4.2$ & $128_{-32}^{+36 \mathrm{a}}$ & ${ }^{82} \mathrm{Zn}$ & $177.9 \pm 2.5$ & $228 \pm 10^{\mathrm{d}}$ \\
\hline
\end{tabular}

${ }^{\mathrm{a}}$ Reference [35].

${ }^{\mathrm{b}}$ Reference [23].

${ }^{\mathrm{c}}$ Reference [21].

${ }^{\mathrm{d}}$ Reference [24]. 
${ }^{78} \mathrm{Ni}$, most of the models included in Fig. 4 of Ref. [16] give a consistent prediction that this neutron shell gap at $Z=28$ is stronger than that at $29 \leq Z \leq 32$. Moreover, the $3.5 \mathrm{MeV}$ gap measured at ${ }^{80} \mathrm{Zn}$ was best reproduced by the finite range liquid drop model (FRDM), which suggested a neutron shell gap over $4.5 \mathrm{MeV}$ in ${ }^{78} \mathrm{Ni}$. This behavior is referred to as the concept of mutual support of magicities [37].

Besides the kink along the $\mathrm{Ni}$ isotopic chain, a large gap can be noticed in Fig. 3 between the half-lives of $\mathrm{Co}$ and $\mathrm{Ni}$ isotopes from $N=44$ to $N=50$. According to the shell model calculation in Ref. [38], the main contribution to the $\beta$ decay ${ }_{27}^{A} \mathrm{Co} \rightarrow{ }_{28}^{A} \mathrm{Ni}$ is either the Gamow-Teller (GT) transition $\nu f_{5 / 2} \rightarrow \pi f_{7 / 2}$ or the FF transition $\nu g_{9 / 2} \rightarrow$ $\pi f_{7 / 2}$. These transitions, however, are greatly hindered in the $\beta$ decay ${ }_{28}^{A} \mathrm{Ni} \rightarrow{ }_{29}^{A} \mathrm{Cu}$ because of the fully occupied $\pi f_{7 / 2}$ SPO inferred from the proton single-particle levels. In the latter case, the proton produced in a $\beta$ decay probably fills the $\pi f_{5 / 2}$ SPO above $\pi f_{7 / 2}$, leading to a reduction of $Q_{\beta}$ value with a magnitude of the $Z=28$ shell-gap energy. Because of the fifth-power relation, a gap is expected between the half-lives of $\mathrm{Co}$ and $\mathrm{Ni}$ isotopes, which agrees with the observation in Fig. 3. The newly measured halflives of ${ }^{76,77} \mathrm{Co}$ follow the decreasing trend with considerable gaps relative to the corresponding $\mathrm{Ni}$ isotones, indicating an almost constant $Z=28$ shell gap without significant quenching up to $N=50$. This conclusion is corroborated by the comparison between the half-lives of $N=50$ isotones and several theoretical calculations shown in Fig. 4. Models include (1) the FRDM + QRPA model [32], in which the half-lives were calculated by the FRDM mass model in conjunction with the GT transition strength given by a quasiparticle random-phase-approximation (QRPA) and the FF transition strength predicted by a statistical gross theory; (2) the KTUY + GT2 model, i.e., the KTUY mass formula [39] combined with the second generation of gross theory (GT2) of the GT and FF transitions [40]; and (3) the shell model taken from Ref. [38], which considers both the GT and FF transitions outside a ${ }^{48} \mathrm{Ca}$ core. As shown in Fig. 4, the step-shape evolution at $Z=28$ is reproduced by all the models. In particular, the shell model provides the best agreement to the experimental data, and it predicts a proton shell-gap energy about $5 \mathrm{MeV}$ between the $\pi f_{7 / 2}$ and $\pi f_{5 / 2}$ SPOs in the calculation.

In summary, the $\beta$-decay half-lives of ${ }^{76,77} \mathrm{Co},{ }^{79,80} \mathrm{Ni}$, and ${ }^{81} \mathrm{Cu}$ are measured in this work. A sudden shortening of half-lives of the nickel isotopes is noticed beyond $N=50$, which is not a strong effect in other isotopic chains from $\mathrm{Cu}$ to $\mathrm{Ga}$. Besides, the half-life gap between $\mathrm{Co}$ and $\mathrm{Ni}$ isotopes, which was previously observed at $N \leq 48$, is found to persist up to $N=50$. With the help of preexisting models, the gross property of half-life evolution suggests a doubly magic character of the neutron-rich nucleus ${ }^{78} \mathrm{Ni}$. Improving half-life calculations based on

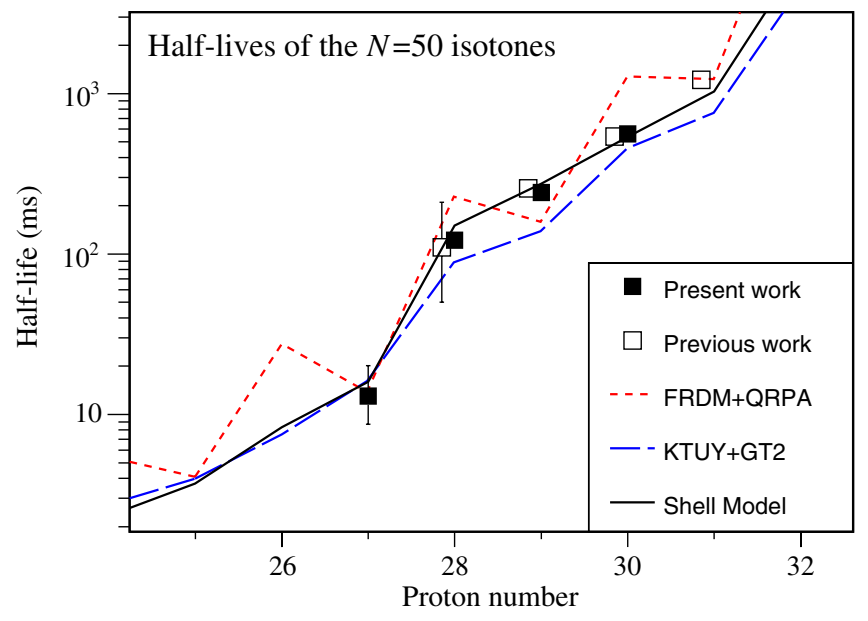

FIG. 4 (color online). Experimental $\beta$-decay half-lives as a function of proton number for the $N=50$ isotones. The solid squares represent the half-lives determined in the present work while the open squares are the previous experimental results taken from either Ref. [21] $\left({ }^{79} \mathrm{Cu}\right)$ or Ref. [35] $\left({ }^{78} \mathrm{Ni},{ }^{80} \mathrm{Zn},{ }^{81} \mathrm{Ga},{ }^{82} \mathrm{Ge}\right.$, and $\left.{ }^{83} \mathrm{As}\right)$. Theoretical models are explained in the main text.

the new results is beyond the scope of this Letter, but will be discussed in a follow-up article. To unambiguously verify the magicity of ${ }^{78} \mathrm{Ni}$ and comprehensively understand the shell evolution in exotic nuclei, future experimental studies such as mass measurement, $\gamma$-ray spectroscopy, and $P_{n}$ measurement are strongly desired and encouraged in this region.

This work was carried out at the RIBF operated by RIKEN Nishina Center, RIKEN and CNS, University of Tokyo. This work was partially supported by KAKENHI (Grants No. 25247045, No. 23.01752, and No. 25800130); U.S. DOE Grant No. DE-FG02-91ER-40609; Spanish Ministerio de Ciencia e Innovación Contracts No. FPA2009-13377-C02 and No. FPA2011-29854-C04; and the Hungarian Scientific Research Fund OTKA Contract No. K100835. The authors acknowledge the EUROBALL Owners Committee for the loan of germanium detectors and the PreSpec Collaboration for the readout electronics of the cluster detectors. Part of the WAS3ABi was supported by the Rare Isotope Science Project which is funded by the Ministry of Education, Science and Technology (MEST) and National Research Foundation (NRF) of Korea. The authors also thank H.Z. Liang, T. Tachibana, and Q. Zhi for valuable discussions.

*xuzy@ ribf.riken.jp

†Present address: National Superconducting Cyclotron Laboratory, Michigan State University, East Lansing, Michigan 48824, USA 
${ }^{\ddagger}$ Present address: Institut für Kernphysik, Technische Universität Darmstadt, Schlossgartenstrasse 9, 64289 Darmstadt, Germany

[1] W. Elsasser, J. Phys. Radium 5, 635 (1934).

[2] M. Goeppert-Mayer, Phys. Rev. 75, 1969 (1949).

[3] O. Haxel, J. H. D. Jensen, and H. E. Suess, Phys. Rev. 75, 1766 (1949).

[4] H. Iwasaki et al., Phys. Lett. B 481, 7 (2000).

[5] T. Motobayashi et al., Phys. Lett. B 346, 9 (1995).

[6] B. Bastin et al., Phys. Rev. Lett. 99, 022503 (2007).

[7] R. Kanungo et al., Phys. Rev. Lett. 102, 152501 (2009).

[8] C. R. Hoffman et al., Phys. Lett. B 672, 17 (2009).

[9] D. Steppenbeck et al., Nature (London) 502, 207 (2013).

[10] S. Franchoo et al., Phys. Rev. Lett. 81, 3100 (1998).

[11] S. Franchoo et al., Phys. Rev. C 64, 054308 (2001).

[12] I. Stefanescu et al., Phys. Rev. Lett. 100, 112502 (2008).

[13] K. Flanagan et al., Phys. Rev. Lett. 103, 142501 (2009).

[14] T. Otsuka, T. Suzuki, R. Fujimoto, H. Grawe, and Y. Akaishi, Phys. Rev. Lett. 95, 232502 (2005).

[15] K. Sieja and F. Nowacki, Phys. Rev. C 81, 061303 (2010).

[16] J. Hakala et al., Phys. Rev. Lett. 101, 052502 (2008).

[17] R. N. Wolf et al., Phys. Rev. Lett. 110, 041101 (2013).

[18] K. Sieja and F. Nowacki, Phys. Rev. C 85, 051301 (2012).

[19] O. Sorlin et al., Phys. Rev. C 47, 2941 (1993).

[20] P. Hosmer et al., Phys. Rev. Lett. 94, 112501 (2005).

[21] P. Hosmer et al., Phys. Rev. C 82, 025806 (2010).

[22] J. M. Daugas et al., Phys. Rev. C 83, 054312 (2011).

[23] C. Mazzocchi et al., Phys. Lett. B 622, 45 (2005).

[24] M. Madurga et al., Phys. Rev. Lett. 109, 112501 (2012).

[25] K. Miernik et al., Phys. Rev. Lett. 111, 132502 (2013).

[26] T. Kubo et al., Prog. Theor. Exp. Phys. 2012, 3C003 (2012).
[27] N. Fukuda, T. Kubo, T. Ohnishi, N. Inabe, H. Takeda, D. Kameda, and H. Suzuki, Nucl. Instrum. Methods Phys. Res., Sect. B 317, 323 (2013).

[28] T. Sumikama et al. (to be published).

[29] S. Nishimura, Prog. Theor. Exp. Phys. 2012, 3C006 (2012).

[30] S. Pietri et al., Nucl. Instrum. Methods Phys. Res., Sect. B 261, 1079 (2007).

[31] P.-A. Söderström et al., Nucl. Instrum. Methods Phys. Res., Sect. B 317, 649 (2013).

[32] P. Möller, B. Pfeiffer, and K.-L. Kratz, Phys. Rev. C 67, 055802 (2003).

[33] I. N. Borzov, Phys. Rev. C 71, 065801 (2005).

[34] Z. Y. Xu, Ph.D. thesis, University of Tokyo, 2014, http:// repository.dl.itc.u-tokyo.ac.jp/index_e.html.

[35] National Nuclear Data Center, http://www.nndc.bnl.gov/ (2013).

[36] X. Zhang, Z. Ren, Q. Zhi, and Q. Zheng, J. Phys. G 34, 2611 (2007).

[37] N. Zeldes, T. Dumitrescu, and H. Köhler, Nucl. Phys. A399, 11 (1983).

[38] Q. Zhi, E. Caurier, J. J. Cuenca-Garcia, K. Langanke, G. Martinez-Pinedo, and K. Sieja, Phys. Rev. C 87, 025803 (2013).

[39] H. Koura, T. Tachibana, M. Uno, and M. Yamada, Prog. Theor. Phys. 113, 305 (2005).

[40] T. Tachibana et al., in Proceedings of ENAM 95: International Conference on Exotic Nuclei and Atomic Masses, Arles, France, 1995, edited by M. de Saint Simon and O. Sorlin (Editions Frontieres, Gif-sur-Yvette, 1995), Vol. 763. 